PROCEEDINGS OF THE

AMERICAN MATHEMATICAL SOCIETY

Volume 127, Number 1, January 1999, Pages 183-194

S 0002-9939(99)04553-0

\title{
AN UNCERTAINTY PRINCIPLE FOR HANKEL TRANSFORMS
}

\author{
MARGIT RÖSLER AND MICHAEL VOIT
}

(Communicated by J. Marshall Ash)

ABstract. There exists a generalized Hankel transform of order $\alpha \geq-1 / 2$ on $\mathbb{R}$, which is based on the eigenfunctions of the Dunkl operator

$$
T_{\alpha} f(x)=f^{\prime}(x)+\left(\alpha+\frac{1}{2}\right) \frac{f(x)-f(-x)}{x}, \quad f \in C^{1}(\mathbb{R}) .
$$

For $\alpha=-1 / 2$ this transform coincides with the usual Fourier transform on $\mathbb{R}$. In this paper the operator $T_{\alpha}$ replaces the usual first derivative in order to obtain a sharp uncertainty principle for generalized Hankel transforms on $\mathbb{R}$. It generalizes the classical Weyl-Heisenberg uncertainty principle for the position and momentum operators on $L^{2}(\mathbb{R})$; moreover, it implies a Weyl-Heisenberg inequality for the classical Hankel transform of arbitrary order $\alpha \geq-1 / 2$ on $[0, \infty[$.

\section{INTRODUCTION}

The classical Heisenberg-Weyl inequality states that for $f \in L^{2}\left(\mathbb{R}^{n}\right)$,

$$
\int_{\mathbb{R}^{n}} x_{j}^{2}|f(x)|^{2} d x \cdot \int_{\mathbb{R}^{n}} \xi_{j}^{2}|\widehat{f}(\xi)|^{2} d \xi \geq \frac{1}{4}\|f\|_{2}^{4} \quad(j=1, \ldots, n)
$$

where

$$
\widehat{f}(\xi)=\frac{1}{(2 \pi)^{n / 2}} \int_{\mathbb{R}^{n}} f(x) e^{-i\langle\xi, x\rangle} d x .
$$

Now suppose that $f \in L^{2}\left(\mathbb{R}^{n}\right)$ is radial, that is $f(x)=f(A x)$ a.e. for all $A \in$ $O(n, \mathbb{R})$. Then there is a unique $F \in L^{2}\left(\left[0, \infty\left[, \omega_{n / 2-1}\right)\right.\right.$ with $f(x)=F\left(\|x\|_{2}\right)$, where for $\alpha \geq-\frac{1}{2}$ the measure $\omega_{\alpha}$ on $[0, \infty[$ is given by

$$
d \omega_{\alpha}(r):=\left(2^{\alpha} \Gamma(\alpha+1)\right)^{-1} r^{2 \alpha+1} d r .
$$

Its normalization assures that $\|f\|_{2}=\|F\|_{2, \omega_{n / 2-1}}$, where $\|\cdot\|_{2}$ is taken with respect to the normalized Lebesgue-measure $(2 \pi)^{-n / 2} d^{n} x$ on $\mathbb{R}^{n}$. For $F \in L^{2}\left(\left[0, \infty\left[, \omega_{\alpha}\right)\right.\right.$ the Hankel transform of $F$ of order $\alpha$ is defined by

$$
\widetilde{F}^{(\alpha)}(\lambda):=\int_{0}^{\infty} j_{\alpha}(\lambda r) F(r) d \omega_{\alpha}(r) \quad(\lambda \geq 0),
$$

Received by the editors October 14, 1996 and, in revised form, May 7, 1997.

1991 Mathematics Subject Classification. Primary 44A15; Secondary 43A62, 26D10, 33 C45.

Key words and phrases. Heisenberg-Weyl inequality, Hankel transform, Dunkl operators, hypergroups.

This paper was partially written at the University of Virginia, Charlottesville, while the first author held a Forschungsstipendium of the DFG.

(C)1999 American Mathematical Society 
with the spherical Bessel functions

$$
j_{\alpha}(z):=\Gamma(\alpha+1)(z / 2)^{-\alpha} J_{\alpha}(z):=\Gamma(\alpha+1) \cdot \sum_{n=0}^{\infty} \frac{(-1)^{n}(z / 2)^{2 n}}{n ! \Gamma(n+\alpha+1)} \quad(z \in \mathbb{C}) .
$$

It is easily checked that for radial $f \in L^{2}\left(\mathbb{R}^{n}\right)$ with $f(x)=F\left(\|x\|_{2}\right)$ the Plancherel transform $\widehat{f}$ is again radial with $\widehat{f}(\xi)=\widetilde{F}^{(n / 2-1)}\left(\|\xi\|_{2}\right)$. Therefore (1.1) for radial functions leads to

$$
\|r F(r)\|_{2} \cdot\left\|\lambda \widetilde{F}^{(n / 2-1)}(\lambda)\right\|_{2} \geq \frac{n}{2}\|F\|_{2}^{2}
$$

for all $F \in L^{2}\left([0, \infty), \omega_{n / 2-1}\right)$, where $\|.\|_{2}$ is taken with respect to $\omega_{n / 2-1}$.

One purpose of this note is to extend the Heisenberg-Weyl uncertainty principle (1.2) to arbitrary indices $\alpha \in \mathbb{R}, \alpha \geq-1 / 2$.

Theorem 1.1. For each $F \in L^{2}\left(\left[0, \infty\left[, \omega_{\alpha}\right)\right.\right.$,

$$
\|x F\|_{2, \omega_{\alpha}} \cdot\left\|\lambda \widetilde{F}^{(\alpha)}\right\|_{2, \omega_{\alpha}} \geq(\alpha+1)\|F\|_{2, \omega_{\alpha}}^{2} .
$$

Moreover, equality holds if and only if $F(x)=d e^{-c x^{2} / 2}$ for some $d \in \mathbb{C}$ and $c>0$.

This theorem emerges as an easy consequence of an uncertainty principle for a generalized Hankel transform of index $\alpha \geq-1 / 2$ on $\mathbb{R}$, where in contrast to the situation on $[0, \infty)$, we have an appropriate generalization of the usual first derivative and thus can follow the classical proof via an inequality for non-commuting selfadjoint operators. This generalization of the usual first derivative is a certain first-order differential-difference operator $T_{\alpha}$ on $\mathbb{R}$, which is known as a Dunkl operator. Such operators have been introduced by Dunkl $[5,6,7]$ in connection with finite reflection groups on Euclidean spaces. They played an essential role in Dunkl's generalization of spherical harmonics and soon led to further development in harmonic analysis (see e.g. Opdam [13] and de Jeu [9]). In particular, there is a generalized Hankel transform on $\mathbb{R}$ (usually called a Dunkl transform) which intertwines $T_{\alpha}$ with the multiplication operator by $x$. It is based on the eigenfunctions of $T_{\alpha}$, which can be expressed in terms of Bessel functions (see Dunkl [7]) and may be considered as generalizations of the usual exponential function. These generalized exponential functions have been studied by Rosenblum [18] in connection with generalized Hermite polynomials and a Bose-like oscillator calculus; for the quantum-mechanical background we refer to the literature cited there, especially to [11], and also to [12]. In fact, the generalized exponential functions lead to an associative convolution structure on $\mathbb{R}$; a detailed investigation of this convolution is given in Rösler [16]. It is quite similar to a hypergroup convolution - up to the lack of preserving positivity - and allows a far-reaching harmonic analysis. (A general reference to hypergroups is the monograph [2].)

The outline of this paper is as follows: In Section 2 basic properties of the generalized Hankel transform on $\mathbb{R}$ and its underlying convolution structure are collected. In Section 3 we introduce generalized Sobolev spaces which provide an appropriate setting for our uncertainty principle. This principle is then stated and proved in Section 4. The functions for which the lower bound is attained are determined there as well. 


\section{A GENERALIZED HANKEL TRANSFORM ON $\mathbb{R}$}

For $\alpha \geq-1 / 2$ we introduce the generalized exponential function

$$
e_{\alpha}(z):=j_{\alpha}(i z)+C_{\alpha} z j_{\alpha+1}(i z) \quad(z \in \mathbb{C}) \quad \text { with } C_{\alpha}=\frac{1}{2(\alpha+1)}
$$

It is holomorphic on $\mathbb{C}$. Notice that in case $\alpha=-1 / 2$ we just have $e_{-1 / 2}(z)=e^{z}$. For $\alpha>-1 / 2$, the function $e_{\alpha}$ has the following integral representation (see [16] or $[18])$ :

$$
e_{\alpha}(z)=M_{\alpha} \int_{-1}^{1} e^{z t}(1-t)^{\alpha-1 / 2}(1+t)^{\alpha+1 / 2} d t
$$

with

$$
M_{\alpha}=\frac{\Gamma(\alpha+1)}{\Gamma(\alpha+1 / 2) \Gamma(1 / 2)} .
$$

Thus $e_{\alpha}$ can be written in terms of the confluent hypergeometric function ${ }_{1} F_{1}$; employing (13.2.2) of Abramowitz and Stegun [1], one obtains

$$
e_{\alpha}(z)=e^{z}{ }_{1} F_{1}(\alpha+1 / 2,2 \alpha+2,-2 z) .
$$

Moreover, (2.1) yields that $\left|e_{\alpha}(z)\right| \leq 1$ for all $z \in i \mathbb{R}$. Rosenblum [18] introduced the functions $e_{\alpha}$ in order to describe the generating functions of the generalized Hermite polynomials on $\mathbb{R}$. He showed that

$$
e^{-z^{2}} e_{\alpha}(2 x z)=\sum_{n=0}^{\infty} H_{n}^{\alpha+1 / 2}(x) \frac{z^{n}}{n !} \quad(x, z \in \mathbb{C}),
$$

where the $H_{n}^{\alpha+1 / 2}, n \in \mathbb{N}_{0}$ are the (suitably normalized) generalized Hermite polynomials of order $\alpha+1 / 2$, being defined as the orthogonal polynomials with respect to the weight $|x|^{2 \alpha+1} e^{-x^{2}}$ on $\mathbb{R}$.

For $\alpha \geq-1 / 2$ and $\lambda \in \mathbb{C}$ the modified Bessel functions $\Psi_{\lambda}^{\alpha}$ on $\mathbb{C}$ are now defined by

$$
\Psi_{\lambda}^{\alpha}(z):=e_{\alpha}(i \lambda z)
$$

The $\Psi_{\lambda}^{\alpha}$ satisfy a product formula on $\mathbb{R}$ which leads to a commutative Banach-*algebra structure on the Banach space $M_{b}(\mathbb{R})$ of all complex bounded Borel measures on $\mathbb{R}$. More precisely, the following is shown in [16]:

Theorem 2.1. Let $\alpha \geq-1 / 2$. Then there is a unique bilinear and separately weak-*-continuous convolution $*_{\alpha}$ on $M_{b}(\mathbb{R})$ such that the product of point measures satisfies

$$
\Psi_{\lambda}^{\alpha}(x) \Psi_{\lambda}^{\alpha}(y)=\int_{\mathbb{R}} \Psi_{\lambda}^{\alpha}(z) d\left(\delta_{x} *_{\alpha} \delta_{y}\right)(z) \quad \text { for } x, y \in \mathbb{R}, \lambda \in \mathbb{C} .
$$

This convolution is associative, commutative, and norm-continuous with

$$
\left(\mu *_{\alpha} \nu\right)(\mathbb{R})=\mu(\mathbb{R}) \cdot \nu(\mathbb{R}) \text { and }\left\|\mu *_{\alpha} \nu\right\| \leq 4 \cdot\|\mu\| \cdot\|\nu\| \text { for } \mu, \nu \in M_{b}(\mathbb{R}) .
$$

Moreover, $\left(M_{b}(\mathbb{R}), *_{\alpha}\right)$ is a commutative Banach-*-algebra with unit $\delta_{0}$, involution $\mu \mapsto \mu^{*}\left(\right.$ where $\mu^{*}(A):=\overline{\mu(-A)}$ for Borel sets $\left.A \subset \mathbb{R}\right)$, and with the norm $\|\mu\|^{\prime}:=$ $\left\|L_{\mu}\right\|$, the operator $L_{\mu}$ on $M_{b}(\mathbb{R})$ being defined by $L_{\mu}(\nu):=\mu * \nu$. 
The convolution $*_{\alpha}$ is given explicitly in [16] as well as in Rosenblum [18], (4.3.1). In fact, it determines a signed hypergroup structure on $\mathbb{R}$. (For a general background on signed hypergroups, we refer to Rösler [14, 15].) At the moment we need no details on this convolution; we just mention that for $x, y \in \mathbb{R}$ the product $\delta_{x} *_{\alpha} \delta_{y}$ is in general not positive and that

$$
\begin{aligned}
\operatorname{supp}\left(\delta_{x} *_{\alpha} \delta_{y}\right)= & {[-|x|-|y|,-|| x|-| y||] } \\
& \cup[|| x|-| y||,|x|+|y|] \text { for } \alpha>-1 / 2, x, y \neq 0 .
\end{aligned}
$$

For $\alpha=-1 / 2$, the convolution $*_{\alpha}$ is just the usual group convolution of $(\mathbb{R},+)$.

Now consider the positive Radon measure

$$
d m_{\alpha}(x)=\frac{1}{2^{\alpha+1} \Gamma(\alpha+1)}|x|^{2 \alpha+1} d x \quad \text { on } \mathbb{R} .
$$

This measure is (up to a multiplicative constant) the unique positive Radon measure on $\mathbb{R}$ which is $*_{\alpha}$-invariant, i.e., for each $f \in C_{c}(\mathbb{R})$ with compact support, we have

$$
m_{\alpha}\left({ }_{x} f\right)=m_{\alpha}(f), \quad \text { where } \quad{ }_{x} f(z):=\int_{\mathbb{R}} f d\left(\delta_{x} *_{\alpha} \delta_{z}\right)
$$

(see Theorem 3.1 of [16]), and notice that the adjoint relation for $m_{\alpha}$ implies (2.3) by Corollary 3.4 of Rösler [14].

When regarded as a subspace of $M_{b}(\mathbb{R})$, the space $L^{1}\left(\mathbb{R}, m_{\alpha}\right)$ becomes a closed *-ideal of $\left(M_{b}(\mathbb{R}), *_{\alpha}\right)$. The multiplicative linear functionals of $L^{1}\left(\mathbb{R}, m_{\alpha}\right)$ are given by

$$
\widetilde{\psi}_{\lambda}^{\alpha}(f):=\int_{\mathbb{R}} f(x) \overline{\Psi_{\lambda}^{\alpha}(x)} d m_{\alpha}(x), \quad \lambda \in \mathbb{R} .
$$

Thus the Gelfand transform is a generalized Hankel transform on $\mathbb{R}$ defined by

$$
L^{1}\left(\mathbb{R}, m_{\alpha}\right) \longmapsto C_{0}(\mathbb{R}), \quad f \longmapsto \widehat{f}^{\alpha} \quad \text { with } \quad \widehat{f}^{\alpha}(\lambda):=\int_{\mathbb{R}} f(x) \overline{\Psi_{\lambda}^{\alpha}(x)} d m_{\alpha}(x) .
$$

There exists a Plancherel theorem (Prop. 3.6 of Rösler [16]), which assures that this transform can be uniquely extended to $L^{2}$-functions and establishes an isometric isomorphism of $L^{2}\left(\mathbb{R}, m_{\alpha}\right)$. For brevity, we shall now always denote the norm of $L^{2}\left(\mathbb{R}, m_{\alpha}\right)$ by $\|\cdot\|_{2}$. Thus

$$
\left\|\widehat{f}^{\alpha}\right\|_{2}=\|f\|_{2} \quad \text { for all } f \in L^{2}\left(\mathbb{R}, m_{\alpha}\right) .
$$

The inverse Hankel and Plancherel transform are defined by

$$
g^{\vee \alpha}(x):=\int_{\mathbb{R}} g(\lambda) \Psi_{\lambda}^{\alpha}(x) d m_{\alpha}(\lambda)
$$

for $g \in L^{1}\left(\mathbb{R}, m_{\alpha}\right)$ and $g \in L^{2}\left(\mathbb{R}, m_{\alpha}\right)$ respectively. If $f \in C(\mathbb{R}) \cap L^{1}\left(\mathbb{R}, m_{\alpha}\right)$ satisfies $\widehat{f}^{\alpha} \in L^{1}\left(\mathbb{R}, m_{\alpha}\right)$, then for all $x \in \mathbb{R}$ we have the $L^{1}$ - inversion formula

$$
f(x)=\left(\widehat{f}^{\alpha}\right)^{\vee \alpha}(x) .
$$

As already indicated, there is a close connection between this convolution structure and the Dunkl transform as studied in de Jeu [9]. In fact, the generalized Hankel transform (2.4) coincides (up to a multiplicative factor) with the Dunkl transform of index $\alpha+1 / 2$ associated with the reflection group $\mathbb{Z}_{2}$ on $\mathbb{R}$. In de Jeu's notation, this transform is defined on $L^{1}\left(\mathbb{R},|x|^{2 \alpha+1} d x\right)$ by

$$
D_{\alpha+1 / 2} f(\lambda)=\int_{\mathbb{R}} f(x) \operatorname{Exp}_{\mathbb{Z}_{2}}(-i \lambda, \alpha+1 / 2, x)|x|^{2 \alpha+1} d x,
$$


with

$$
\begin{aligned}
\operatorname{Exp}_{\mathbb{Z}_{2}} & (-i \lambda, \alpha+1 / 2, x) \\
& =\Gamma(\alpha+1)\left(\frac{|x \lambda|}{2}\right)^{-\alpha}\left(J_{\alpha}(|x \lambda|)-i \operatorname{sgn}(x \lambda) J_{\alpha+1}(|x \lambda|)\right)=\overline{\Psi_{\lambda}^{\alpha}}(x)
\end{aligned}
$$

(see Dunkl [7], Section 4 and Remark 4.24 in de Jeu [9]). It was observed by Dunkl that the $\Psi_{\lambda}^{\alpha}, \lambda \in \mathbb{C}$, are eigenfunctions of the first-order differential-difference operator

$$
T_{\alpha} f(x)=f^{\prime}(x)+\left(\alpha+\frac{1}{2}\right) \cdot \frac{f(x)-f(-x)}{x}, \quad f \in C^{1}(\mathbb{R})
$$

satisfying

$$
T_{\alpha}\left(\Psi_{\lambda}^{\alpha}\right)=i \lambda \cdot \Psi_{\lambda}^{\alpha}
$$

\section{The Sobolev spaces $H_{2}^{\alpha}(\mathbb{R})$}

For our purpose of an uncertainty principle involving $T_{\alpha}$ it is useful to introduce the generalized Sobolev space

$$
H_{2}^{\alpha}(\mathbb{R}):=\left\{f \in L^{2}\left(\mathbb{R}, m_{\alpha}\right): \lambda \widehat{f}^{\alpha}(\lambda) \in L^{2}\left(\mathbb{R}, m_{\alpha}\right)\right\},
$$

equipped with the norm

$$
\left\|f \mid H_{2}^{\alpha}\right\|:=\left(\|f\|_{2}^{2}+\left\|\lambda \widehat{f}^{\alpha}(\lambda)\right\|_{2}^{2}\right)^{1 / 2} .
$$

(As before, $\|\cdot\|_{2}$ is taken with respect to $m_{\alpha}$.) The space $H_{2}^{\alpha}(\mathbb{R})$ is isomorphic with the Hilbert space $L^{2}\left(\mathbb{R},\left(1+\lambda^{2}\right) d m_{\alpha}(\lambda)\right)$ via the Plancherel transform $f \mapsto \widehat{f}^{\alpha}$. According to Corollary 4.22 of de Jeu [9], this transform provides a homeomorphism of the Schwartz space $\mathcal{S}(\mathbb{R})$ of rapidly decreasing functions on $\mathbb{R}$. It is easily checked that $\mathcal{S}(\mathbb{R})$ is dense in $L^{2}\left(\mathbb{R},\left(1+\lambda^{2}\right) d m_{\alpha}(\lambda)\right)$. As a consequence, $\mathcal{S}(\mathbb{R})$ is dense in $H_{2}^{\alpha}(\mathbb{R})$ as well. For $f \in \mathcal{S}(\mathbb{R})$ and $g \in C_{b}^{1}(\mathbb{R})$ we have

$$
\int_{\mathbb{R}}\left(T_{\alpha} f\right) g d m_{\alpha}=-\int_{\mathbb{R}} f\left(T_{\alpha} g\right) d m_{\alpha}
$$

and

$$
\left(T_{\alpha} f\right)^{\wedge \alpha}(\lambda)=i \lambda \cdot \widehat{f}^{\alpha}(\lambda)
$$

(see Dunkl [7] and the weaker versions of de Jeu [9], Lemma 4.6). The operator $T_{\alpha}$ extends canonically to $H_{2}^{\alpha}(\mathbb{R})$ by setting $T_{\alpha} f:=\left(i \lambda \cdot \widehat{f}^{\alpha}\right)^{\vee \alpha}$ for $f \in H_{2}^{\alpha}(\mathbb{R})$. Thus $i T_{\alpha}$ is a self-adjoint operator on $L^{2}\left(\mathbb{R}, m_{\alpha}\right)$ with domain $\mathcal{D}\left(i T_{\alpha}\right)=\mathcal{D}\left(T_{\alpha}\right)=H_{2}^{\alpha}(\mathbb{R})$.

Later on, we shall need information on the asymptotic behaviour of functions from $H_{2}^{\alpha}(\mathbb{R})$ near 0 . According to Sobolev's lemma, functions belonging to the classical Sobolev space $H_{2}(\mathbb{R})=H_{2}^{-1 / 2}(\mathbb{R})$ always have a representative which is continuous on $\mathbb{R}$. This is in general not true for functions from $H_{2}^{\alpha}(\mathbb{R})$ with $\alpha \geq 0$. The following lemma gives sharp estimates for their asymptotic behaviour near 0 and shows in particular that for each $f \in H_{2}^{\alpha}(\mathbb{R})$ there exists a representative of $f$ which is at least continuous on $\mathbb{R} \backslash\{0\}$.

Lemma 3.1. Let $f \in H_{2}^{\alpha}(\mathbb{R})$. Then there exists an $L^{2}$-representative of $f$ such that

$$
|f(x)| \leq C_{\alpha} \cdot\left\|f \mid H_{2}^{\alpha}\right\| \cdot R_{\alpha}(x)
$$


for $x \neq 0$, where $C_{\alpha}>0$ is a constant independent of $f$ and

$$
R_{\alpha}(x):= \begin{cases}|x|^{-\alpha} & \text { if } \alpha>0 ; \\ |\ln | x||^{1 / 2} & \text { if } \alpha=0 ; \\ 1 & \text { if } \alpha \in[-1 / 2,0[.\end{cases}
$$

Moreover, this $L^{2}$-representative of $f$ is continuous on $\mathbb{R} \backslash\{0\}$ for any $\alpha \geq-1 / 2$ and continuous on $\mathbb{R}$ if $\alpha \in[-1 / 2,0[$.

Proof. The definition of the characters $\Psi_{\lambda}^{\alpha}$ and the asymptotic expansion for Bessel functions (see (9.2.1) of Abramowitz and Stegun [1]) ensure that

$$
\Psi_{\lambda}^{\alpha}(x)=O\left(|\lambda x|^{-(\alpha+1 / 2)}\right) \quad \text { for } \quad|\lambda x| \rightarrow \infty .
$$

Moreover, as noted in Section 2, we have $\left|\Psi_{\lambda}^{\alpha}(x)\right| \leq 1$ for $\lambda, x \in \mathbb{R}$. Hence there exist constants $C_{i}$ such that for all $x \neq 0$

$$
\begin{aligned}
\int_{\mathbb{R}} \mid \widehat{f}^{\alpha}(\lambda) & \Psi_{\lambda}^{\alpha}(x)\left|d m_{\alpha}(\lambda) \leq C_{1} \int_{\mathbb{R}}\right| \widehat{f}^{\alpha}(\lambda) \mid \cdot \min \left(1,|\lambda x|^{-(\alpha+1 / 2)}\right) d m_{\alpha}(\lambda) \\
& \leq C_{1} \int_{-1 /|x|}^{1 /|x|}\left|\widehat{f}^{\alpha}(\lambda)\right| d m_{\alpha}(\lambda)+\frac{C_{2}}{|x|^{\alpha+1 / 2}} \int_{\{|\lambda x| \geq 1\}}\left|\widehat{f}^{\alpha}(\lambda)\right||\lambda|^{\alpha+1 / 2} d \lambda \\
& \leq C_{3}\left(\int_{-1 /|x|}^{1 /|x|}\left|\widehat{f}^{\alpha}(\lambda)\right|^{2}\left(1+\lambda^{2}\right) d m_{\alpha}(\lambda) \cdot \int_{-1 /|x|}^{1 /|x|} \frac{|\lambda|^{2 \alpha+1}}{1+\lambda^{2}} d \lambda\right)^{1 / 2} \\
& +\frac{C_{4}}{|x|^{\alpha+1 / 2}}\left(\int_{\mathbb{R}}\left|\widehat{f}^{\alpha}(\lambda)\right|^{2}\left(1+\lambda^{2}\right) d m_{\alpha}(\lambda) \cdot \int_{\{|\lambda x| \geq 1\}} \frac{1}{1+\lambda^{2}} d \lambda\right)^{1 / 2} \\
& \leq C_{5} \cdot R_{\alpha}(x) \cdot\left\|f \mid H_{2}^{\alpha}\right\| .
\end{aligned}
$$

Now take a sequence $\left(f_{n}\right) \subset \mathcal{S}(\mathbb{R})$ with $f_{n} \rightarrow f$ in $H_{2}^{\alpha}(\mathbb{R})$. Applying (3.4) to the differences $f-f_{n}$, we see that $\left(f_{n}\right)$ converges locally uniformly on $\mathbb{R} \backslash\{0\}$ to $\varphi(x):=\int_{\mathbb{R}} \widehat{f}^{\alpha}(\lambda) \Psi_{\lambda}^{\alpha}(x) d m_{\alpha}(\lambda)$. Hence $\varphi$ is a continuous representative of $f$ on $\mathbb{R} \backslash\{0\}$, and (3.4) shows that it satisfies (3.3). Finally, if $\alpha<0$, then the $f_{n}$ converge uniformly on $\mathbb{R} \backslash\{0\}$ and hence on $\mathbb{R}$ as well. This yields a continuous extension of $\varphi$ to 0 .

Remark 3.2. The estimates of Lemma 3.1 are sharp in the following sense: If $\alpha>0$, then for any exponent $\beta \in] 0, \alpha\left[\right.$ there exist functions $f \in H_{2}^{\alpha}(\mathbb{R})$ with $f(x) \sim|x|^{-\beta}$ for $x \rightarrow 0$; if $\alpha=0$, then for any exponent $\beta \in] 0,1 / 2[$ there exist functions $f \in H_{2}^{0}(\mathbb{R})$ with $f(x) \sim|\ln | x||^{\beta}$ for $x \rightarrow 0$.

In fact, if $\alpha>0$, then one may choose $f:=g^{\vee \alpha}$ with $g \in L^{2}\left(\mathbb{R}, m_{\alpha}\right)$ being defined by $g(\lambda)=|\lambda|^{\beta-2 \alpha-2}$ for $|\lambda| \geq 1$ and $g(\lambda)=1$ for $|\lambda|<1$. (Note that $\left.g \notin L^{1}\left(\mathbb{R}, m_{\alpha}\right)\right)$. Then for $x \neq 0$, one has

$$
f(x)=\int_{\mathbb{R}} g(\lambda) \Psi_{\lambda}^{\alpha}(x) d m_{\alpha}(\lambda)=\int_{0}^{1} j_{\alpha}(\lambda x) d \omega_{\alpha}(\lambda)+\int_{1}^{\infty} \lambda^{\beta-2 \alpha-2} j_{\alpha}(\lambda x) d \omega_{\alpha}(\lambda) .
$$

The first summand is obviously bounded with $x \rightarrow 0$, while the second one equals

$$
\frac{C_{1}}{|x|^{\beta}} \int_{|x|}^{\infty} u^{\beta-1} j_{\alpha}(u) d u \sim C_{2}|x|^{-\beta} \quad \text { for } x \rightarrow 0
$$

with some constants $C_{1}, C_{2} \in \mathbb{R}$. (Note that by the asymptotics of $j_{\alpha}(u)$ with $u \rightarrow \infty$, the integral $\int_{0}^{\infty} u^{\beta-1} j_{\alpha}(u) d u$ converges.) 
If $\alpha=0$, then choose $f=g^{\vee 0}$ with $g \in L^{2}\left(\mathbb{R}, m_{0}\right)$ being defined by $g(\lambda)=$ $|\lambda|^{-2}(\ln |\lambda|)^{\beta-1}$ for $|\lambda| \geq e$ and $g(\lambda)=1$ for $|\lambda|<e$. For small $|x| \neq 0$ we now obtain, with certain functions $C_{i}(x)$ which are bounded when $x \rightarrow 0$,

$$
\begin{aligned}
f(x) & =\int_{\mathbb{R}} g(\lambda) \Psi_{\lambda}^{0}(x) d m_{0}(\lambda)=C_{1}(x)+\int_{e}^{\infty} \frac{(\ln \lambda)^{\beta-1}}{\lambda^{2}} j_{0}(\lambda|x|) \lambda d \lambda \\
& =C_{2}(x)+\int_{e|x|}^{1} \frac{1}{u}\left[\ln \left(\frac{u}{|x|}\right)\right]^{\beta-1} j_{0}(u) d u .
\end{aligned}
$$

As $j_{0}(u)=1+O\left(u^{2}\right)$ with $u \rightarrow 0$, this equals

$C_{3}(x)+\int_{e|x|}^{1} \frac{1}{u}\left[\ln \left(\frac{u}{|x|}\right)\right]^{\beta-1} d u=C_{3}(x)+\left.\frac{1}{\beta}\left[\ln \left(\frac{u}{|x|}\right)\right]^{\beta}\right|_{e|x|} ^{1}=C_{4}(x)+\frac{1}{\beta}|\ln | x||^{\beta}$ as claimed.

Remark 3.3. Results related to those of this section can also be found in [21] and references cited there.

\section{The UnCERTAinty PRINCIPle For Hankel transforms on $\mathbb{R}$}

We shall use the following notion of variance: Let $Q$ denote the multiplication operator on $L^{2}\left(\mathbb{R}, m_{\alpha}\right)$ defined by $Q f(x)=x f(x)$; its domain is

$$
\mathcal{D}(Q)=\left\{f \in L^{2}\left(\mathbb{R}, m_{\alpha}\right): x f \in L^{2}\left(\mathbb{R}, m_{\alpha}\right)\right\} .
$$

Suppose $f \in \mathcal{D}(Q)$ with $\|f\|_{2}=1$. Then, denoting the scalar product on $L^{2}\left(\mathbb{R}, m_{\alpha}\right)$ by $\langle$,$\rangle , we define the \alpha$-variance of $f$ by

$$
\operatorname{var}_{\alpha}(f):=\|x f\|_{2}^{2}-\langle x f, f\rangle^{2}=\|(x-\langle x f, f\rangle) f\|_{2}^{2} .
$$

In case $f \in H_{2}^{\alpha}(\mathbb{R})$ it is seen from (3.2) that

$$
\operatorname{var}_{\alpha}\left(\widehat{f}^{\alpha}\right)=\left\|\left(T_{\alpha}-\left\langle T_{\alpha} f, f\right\rangle\right) f\right\|_{2}^{2} .
$$

Finally, for a function $f: \mathbb{R} \rightarrow \mathbb{C}$ denote by

$$
f_{e}(x)=\frac{1}{2}(f(x)+f(-x)), \quad f_{o}(x)=\frac{1}{2}(f(x)-f(-x))
$$

its even and odd part respectively. Our main theorem is then as follows:

Theorem 4.1. Let $f \in \mathcal{D}\left(T_{\alpha}\right) \cap \mathcal{D}(Q)$ with $\|f\|_{2}=1$. Then

$$
\operatorname{var}_{\alpha}(f) \cdot \operatorname{var}_{\alpha}\left(\widehat{f}^{\alpha}\right) \geq\left(\left(\alpha+\frac{1}{2}\right)\left(\left\|f_{e}\right\|_{2}^{2}-\left\|f_{o}\right\|_{2}^{2}\right)+\frac{1}{2}\right)^{2} .
$$

Moreover, equality holds if and only if $f$ has the form

$$
f_{(b, c)}(x):=d_{(b, c)} \cdot e^{-c x^{2} / 2} \cdot \Psi_{b}^{\alpha}(x) \quad \text { with } b \in \mathbb{C}, c>0,
$$

and with a suitable normalization constant $d_{(b, c)}>0$.

Remarks 4.2. 1. For $\alpha=-1 / 2$, we regain the classical uncertainty principle:

$$
\operatorname{var}(f) \cdot \operatorname{var}(\widehat{f}) \geq \frac{1}{4},
$$

with equality if and only if $f$ is a Gaussian density of the form

$$
f(x)=\left(\frac{c}{\pi}\right)^{1 / 4} \cdot e^{-c x^{2}+i b x} \quad \text { with some } b \in \mathbb{C} \text { and } c>0 .
$$


2. The usual Hankel transform of $L^{2}\left(\left[0, \infty\left[, \omega_{\alpha}\right)\right.\right.$-functions is connected with the generalized Hankel transform of even $L^{2}\left(\mathbb{R}, m_{\alpha}\right)$-functions in an obvious way: for $f \in L^{2}\left(\left[0, \infty\left[, \omega_{\alpha}\right)\right.\right.$ and $F \in L^{2}\left(\mathbb{R}, m_{\alpha}\right)$ being defined by $F(x)=f(|x|)$, we have $\widehat{F}^{\alpha}(\lambda)=\tilde{f}^{\alpha}(|\lambda|)$. Therefore, Theorem 4.1 immediately implies Theorem 1.1.

3. The normalization constants $d_{(b, c)}$ will be computed explicitly in Lemma 4.3. In particular it will turn out that for $b \in \mathbb{C}$ and $c>0$ the even and odd parts of the "optimal" functions $f_{(b, c)}$ satisfy

$$
\left\|f_{(b, c), e}\right\|_{2} \geq\left\|f_{(b, c), o}\right\|_{2}
$$

and

$$
\left\|f_{(b, c), e}\right\|_{2}-\left\|f_{(b, c), o}\right\|_{2} \rightarrow 0 \text { for }|b| \rightarrow \infty,
$$

which means that for fixed $\alpha>-1 / 2$, the lower bound $1 / 4$ of the classical uncertainty principle is attained asymptotically with $|b| \rightarrow \infty$.

4. There exist further uncertainty principles for $(\mathbb{R},+)$ which can be extended to the convolution structures above. Here we only mention a principle due to Strichartz [19], as well as the $\epsilon-\delta$-concentration uncertainty principle of Donoho and Stark [4]. The latter may be derived here in the same way as described in Voit [20]; it is also covered by an $\epsilon-\delta$-concentration principle for certain integral operators proved by de Jeu [10].

5 . We expect that the results given in this paper can be extended to further classes of Dunkl transforms related to finite reflection groups acting on $\mathbb{R}^{n}$.

Proof of Theorem 4.1. 1. Proof of inequality (4.2) in the case that $f \in \mathcal{S}(\mathbb{R})$. A short calculation shows that

$$
\left[T_{\alpha}, Q\right](f)=\left(T_{\alpha} Q-Q T_{\alpha}\right)(f)=(2 \alpha+2) f_{e}-2 \alpha f_{o} .
$$

The Cauchy-Schwarz inequality and the anti-symmetry of $T_{\alpha}$ ensure that

$$
\begin{aligned}
\operatorname{var}_{\alpha}(f)^{1 / 2} \cdot \operatorname{var}_{\alpha}\left(\widehat{f}^{\alpha}\right)^{1 / 2}=\|(Q-\langle Q f, f\rangle) f\|_{2} \cdot\left\|\left(T_{\alpha}-\left\langle T_{\alpha} f, f\right\rangle\right) f\right\|_{2} \\
\quad \geq \frac{1}{2}\left|\left\langle\left[T_{\alpha}, Q\right] f, f\right\rangle\right|=\frac{1}{2}\left|\left\langle(2 \alpha+2) f_{e}-2 \alpha f_{o}, f_{e}+f_{o}\right\rangle\right| \\
\quad=(\alpha+1)\left\|f_{e}\right\|_{2}^{2}-\alpha\left\|f_{o}\right\|_{2}^{2}=\left(\alpha+\frac{1}{2}\right)\left(\left\|f_{e}\right\|_{2}^{2}-\left\|f_{o}\right\|_{2}^{2}\right)+\frac{1}{2} .
\end{aligned}
$$

2. Extension of inequality (4.2) to its maximal range $\mathcal{D}\left(T_{\alpha}\right) \cap \mathcal{D}(Q)$. This extension affords an approximation process, for which we keep close to the method used in Dym and McKean [8] for the classical case. Involving again the Cauchy-Schwarz inequality and the anti-symmetry of $T_{\alpha}$, a little more careful argumentation than before yields

$$
\begin{aligned}
\operatorname{var}_{\alpha} & (f)^{1 / 2} \cdot \operatorname{var}_{\alpha}\left(\widehat{f}^{\alpha}\right)^{1 / 2} \\
& \geq\left|\left\langle(x-\langle x f, f\rangle) f,\left(T_{\alpha}-\left\langle T_{\alpha} f, f\right\rangle\right) f\right\rangle\right| \\
& =\left|\left\langle x f, T_{\alpha} f\right\rangle-\langle x f, f\rangle \cdot\left\langle f, T_{\alpha} f\right\rangle\right| \\
& \geq\left|\operatorname{Re}\left(\left\langle x f, T_{\alpha} f\right\rangle-\langle x f, f\rangle \cdot\left\langle f, T_{\alpha} f\right\rangle\right)\right| \\
& =\left|\operatorname{Re}\left\langle x f, T_{\alpha} f\right\rangle\right| .
\end{aligned}
$$

Now choose a sequence $\left(f_{n}\right) \subset \mathcal{S}(\mathbb{R})$ with $f_{n} \rightarrow f$ in $H_{2}^{\alpha}(\mathbb{R})$, and define $g_{l} \in$ $C_{c}^{1}(\mathbb{R}), l \in \mathbb{N}$, with $g_{l}(x)=x$ on $I_{l}:=[-l,-1 / l] \cup[1 / l, l]$ and $g_{l}(x)=0$ on $\mathbb{R} \backslash I_{l+1}$ 
such that $\left|T_{\alpha}\left(g_{l}\right)(x)\right| \leq M l$ for all $l \in \mathbb{N}$, where $M>0$ is a constant. Then

$$
\begin{aligned}
\operatorname{Re}\left\langle x f, T_{\alpha} f\right\rangle & =\lim _{l \rightarrow \infty} \operatorname{Re}\left\langle g_{l} f, T_{\alpha} f\right\rangle=\lim _{l \rightarrow \infty} \lim _{n \rightarrow \infty} \operatorname{Re}\left\langle g_{l} f_{n}, T_{\alpha} f_{n}\right\rangle \\
& =\lim _{l \rightarrow \infty} \lim _{n \rightarrow \infty}\left\langle g_{l}, \operatorname{Re}\left(\overline{f_{n}} T_{\alpha} f_{n}\right)\right\rangle
\end{aligned}
$$

For $h \in C^{1}(\mathbb{R})$ we can write

$$
\begin{aligned}
2 \operatorname{Re}\left(\bar{h} T_{\alpha} h\right)(x) & =T_{\alpha}\left(|h|^{2}\right)(x)+\frac{\alpha+1 / 2}{x}(2 \operatorname{Re}((h(x)-h(-x)) \overline{h(x)}) \\
& \left.-|h(x)|^{2}+|h(-x)|^{2}\right)=T_{\alpha}\left(|h|^{2}\right)(x)+\frac{4 \alpha+2}{x}\left|h_{o}(x)\right|^{2} .
\end{aligned}
$$

We thus obtain

$$
\begin{aligned}
\operatorname{Re}\left\langle x f, T_{\alpha} f\right\rangle & =\lim _{l \rightarrow \infty} \lim _{n \rightarrow \infty}\left(\left\langle g_{l}, \frac{1}{2} T_{\alpha}\left(\left|f_{n}\right|^{2}\right)\right\rangle+(2 \alpha+1)\left\langle\frac{1}{x} g_{l}(x),\left|f_{n, o}\right|^{2}\right\rangle\right) \\
& =\frac{1}{2} \lim _{l \rightarrow \infty} \lim _{n \rightarrow \infty}-\left\langle T_{\alpha} g_{l},\left|f_{n}\right|^{2}\right\rangle+(2 \alpha+1)\left\|f_{o}\right\|_{2}^{2} \\
& =-\frac{1}{2} \lim _{l \rightarrow \infty}\left\langle T_{\alpha} g_{l},|f|^{2}\right\rangle+(2 \alpha+1)\left\|f_{o}\right\|_{2}^{2} .
\end{aligned}
$$

Now observe that $T_{\alpha}\left(g_{l}\right)=2(\alpha+1)$ on $I_{l}$ and $T_{\alpha}\left(g_{l}\right)=0$ on $\mathbb{R} \backslash I_{l+1}$. Moreover, since $f \in L^{2}\left(\mathbb{R}, m_{\alpha}\right)$, there is a subsequence of $\left(l \cdot \int_{I_{l+1} \backslash I_{l}}|f|^{2} d m_{\alpha}\right)_{l \geq 1}$ which tends to 0 ; this shows that $\lim _{l \rightarrow \infty} \int_{I_{l+1} \backslash I_{l}}\left(T_{\alpha} g_{l}\right)|f|^{2} d m_{\alpha}=0$, by the assumption on $\left(T_{\alpha} g_{l}\right)_{l \in \mathbb{N}}$. Together we obtain

$$
\lim _{l \rightarrow \infty}\left\langle T_{\alpha} g_{l},|f|^{2}\right\rangle=2(\alpha+1)\|f\|_{2}^{2}=2(\alpha+1)
$$

and therefore

$$
\operatorname{Re}\left\langle x f, T_{\alpha} f\right\rangle=-(\alpha+1)+(2 \alpha+1)\left\|f_{o}\right\|_{2}^{2}=-\left(\alpha+\frac{1}{2}\right)\left(\left\|f_{e}\right\|_{2}^{2}-\left\|f_{o}\right\|_{2}^{2}\right)-\frac{1}{2} .
$$

This yields the assertion.

Proof of the case when the lower bound is attained. The key step in the proof above is the application of the Cauchy-Schwarz inequality in (4.5) and then taking the real part. This means that the bound is attained if and only if

$$
(-c) \cdot(x-\langle x f, f\rangle) f=\left(T_{\alpha}-\left\langle T_{\alpha} f, f\right\rangle\right) f \quad \text { a.e. }
$$

for some $c \in \mathbb{R}$. We first check that each solution $f \in H_{2}^{\alpha}(\mathbb{R})$ of (4.7) belongs to $C^{\infty}(\mathbb{R} \backslash\{0\})$ and satisfies

$$
T_{\alpha} f(x)=f^{\prime}(x)+\left(\alpha+\frac{1}{2}\right) \frac{f(x)-f(-x)}{x} \quad \text { for } x \neq 0 .
$$


For this we choose functions $f_{n} \in \mathcal{S}(\mathbb{R})$ with $f_{n} \rightarrow f$ in $H_{2}^{\alpha}(\mathbb{R})$. Then for $x>0$ and fixed $\delta>0$ we have

$$
\begin{aligned}
\int_{\delta}^{x} & \left(T_{\alpha} f\right)(\xi) \cdot \xi^{2 \alpha+1} d \xi=\lim _{n \rightarrow \infty} \int_{\delta}^{x}\left(T_{\alpha} f_{n}\right)(\xi) \cdot \xi^{2 \alpha+1} d \xi \\
& =\lim _{n \rightarrow \infty}\left(\int_{\delta}^{x} f_{n}^{\prime}(\xi) \cdot \xi^{2 \alpha+1} d \xi+\left(\alpha+\frac{1}{2}\right) \int_{\delta}^{x}\left(f_{n}(\xi)-f_{n}(-\xi)\right) \cdot \xi^{2 \alpha} d \xi\right) \\
& =\lim _{n \rightarrow \infty}\left(x^{2 \alpha+1} f_{n}(x)-\delta^{2 \alpha+1} f_{n}(\delta)-\left(\alpha+\frac{1}{2}\right) \int_{\delta}^{x}\left(f_{n}(\xi)+f_{n}(-\xi)\right) \cdot \xi^{2 \alpha} d \xi\right) \\
& =x^{2 \alpha+1} f(x)-\delta^{2 \alpha+1} f(\delta)-\left(\alpha+\frac{1}{2}\right) \int_{\delta}^{x}(f(\xi)+f(-\xi)) \cdot \xi^{2 \alpha} d \xi .
\end{aligned}
$$

Substitution of (4.7) in the integral on the left yields that $f$ is infinitely often differentiable on $] 0, \infty[$ and, by the same argument, also on $]-\infty, 0[$. Finally, differentiation of (4.9) shows that (4.8) is indeed satisfied.

Thus we are led to solve the differential-difference equation

$$
T_{\alpha} f(x)=(b-c x) f(x) \quad(c \in \mathbb{R}, b \in \mathbb{C})
$$

with $f \in C^{\infty}(\mathbb{R} \backslash\{0\})$ (where in fact, $b$ and $c$ depend on $f$ ). First note that for $f \in C^{1}(\mathbb{R} \backslash\{0\})$ and even $g \in C^{1}(\mathbb{R} \backslash\{0\})$ the following product formula holds:

$$
T_{\alpha}(f g)=\left(T_{\alpha} f\right) \cdot g+f \cdot\left(T_{\alpha} g\right) .
$$

It follows that $f \in C^{\infty}(\mathbb{R} \backslash\{0\})$ solves (4.10) exactly if $F(x):=e^{c x^{2} / 2} f(x)$ satisfies

$$
T_{\alpha} F=b F .
$$

Insert $-x$ into (4.11) and form the sum and difference with (4.11). This leads to

$$
F_{o}^{\prime}(x)+(2 \alpha+1) \frac{F_{o}(x)}{x}=b F_{e}(x) ; \quad F_{e}^{\prime}(x)=b F_{o}(x) .
$$

We may assume that $b \neq 0$, because for $b=0$ explicit solution of (4.12) would yield that $\left|F_{o}\right|$ is of the form $C \cdot|x|^{-(2 \alpha+1)}$, which contradicts Lemma 3.1. Differentiation of the second equality in (4.12) shows that $F_{e}$ satisfies the modified Bessel equation

$$
w^{\prime \prime}+\frac{2 \alpha+1}{x} w^{\prime}=b^{2} w \quad \text { on } \mathbb{R} \backslash\{0\} .
$$

Independent solutions of (4.13) are given by the holomorphic solution $w_{1}(x):=$ $j_{\alpha}(i b x)$ as well as by

$$
w_{2}(x):= \begin{cases}|x|^{-2 \alpha} \cdot j_{-\alpha}(i b x) & \text { for } \alpha \geq-1 / 2, \alpha \neq 0,1,2, \ldots \\ |x|^{-\alpha} \cdot Y_{\alpha}(i b|x|) & \text { for } \alpha=0,1,2, \ldots\end{cases}
$$

where $Y_{\alpha}$ is the Bessel function of the second kind. If $\alpha>0$, then $w_{2}(x) \sim C \cdot|x|^{-2 \alpha}$. As $f_{e}(x)=O\left(|x|^{-\alpha}\right)$ for $x \rightarrow 0$ by Lemma 3.1, it follows that $F_{e}$ must be a multiple of $w_{1}$. If $\alpha=0$, then the same conclusion holds, because in this case $w_{2}(x) \sim$ $C \cdot \ln |x|$, while $f_{e}(x)=O\left(|\ln (|x|)|^{1 / 2}\right)$ for $x \rightarrow 0$. Finally, if for $\left.\alpha \in\right]-1 / 2,0[$ a $w_{2}$-part would appear in $F_{e}$, then (4.12) would imply that $\left|F_{o}(x)\right| \sim C \cdot|x|^{-(2 \alpha+1)}$, which would again contradict Lemma 3.1.

Together we have shown that

$$
f_{e}(x)=d \cdot e^{-c x^{2} / 2} j_{\alpha}(i b x) \quad \text { with } d \in \mathbb{C} .
$$


The second formula of (4.12) now yields

$$
f_{o}(x)=\frac{d}{b} \cdot e^{-c x^{2} / 2} \cdot \frac{d}{d x}\left(j_{\alpha}(i b x)\right)=d e^{-c x^{2} / 2} \cdot \frac{b x}{2(\alpha+1)} \cdot j_{\alpha+1}(i b x)
$$

and therefore

$$
f(x)=d \cdot e^{-c x^{2} / 2} \cdot \Psi_{-i b}^{\alpha}(x) \quad \text { for } c>0, b, d \in \mathbb{C} .
$$

Conversely, if $f$ has the form (4.14) with $\|f\|_{2}=1$, then it is clear that (4.7) holds, i.e., that the bound is in fact attained.

We still have to compute the normalization constants $d_{(b, c)}$ of Theorem 4.1 and to verify the statements of Remark 4.2. But these are immediate consequences of formula (3.3.4) in Rosenblum [18], which says that for all $c>0$ and $b, \lambda \in \mathbb{C}$,

$$
\int_{\mathbb{R}} e^{-c x^{2} / 2} \cdot \Psi_{\lambda}^{\alpha}(x) \cdot \Psi_{b}^{\alpha}(x) d m_{\alpha}(x)=\frac{1}{c^{\alpha+1}} \cdot e^{-\left(b^{2}+\lambda^{2}\right) /(2 c)} \cdot e_{\alpha}(-b \lambda / c) .
$$

Together with the identity $\overline{\Psi_{\lambda}^{\alpha}(x)}=\Psi_{-\bar{\lambda}}^{\alpha}(x)$ for $x \in \mathbb{R}$, formula (4.15) leads to

Lemma 4.3. The functions $\widetilde{f}_{(b, c)}:=e^{-c x^{2} / 2} \cdot \Psi_{b}^{\alpha}(x)(b \in \mathbb{C}, c>0)$ satisfy

(1) $\int_{\mathbb{R}}\left|\tilde{f}_{(b, c)}\right|^{2} d m_{\alpha}=\frac{1}{(2 c)^{\alpha+1}} \cdot e^{-\operatorname{Re} b^{2} /(2 c)} \cdot e_{\alpha}\left(|b|^{2} /(2 c)\right)$.

(2) $\int_{\mathbb{R}}\left|\widetilde{f}_{(b, c), e}\right|^{2} d m_{\alpha}=\frac{1}{2(2 c)^{\alpha+1}} \cdot e^{-\operatorname{Re} b^{2} /(2 c)} \cdot\left(e_{\alpha}\left(|b|^{2} /(2 c)\right)+e_{\alpha}\left(-|b|^{2} /(2 c)\right)\right)$.

(3) $\int_{\mathbb{R}}\left|\widetilde{f}_{(b, c), o}\right|^{2} d m_{\alpha}=\frac{1}{2(2 c)^{\alpha+1}} \cdot e^{-\operatorname{Re} b^{2} /(2 c)} \cdot\left(e_{\alpha}\left(|b|^{2} /(2 c)\right)-e_{\alpha}\left(-|b|^{2} /(2 c)\right)\right)$.

From the integral representation $(2.1)$ it is seen that $e_{\alpha}\left(-|b|^{2} /(2 c)\right)$ is nonnegative and tends to 0 with $|b| \rightarrow \infty$. Thus (4.3) and (4.4) are immediate consequences of Lemma 4.3, and the proof of our statements in Section 4 is now complete.

\section{NOTE ADDED IN PROOF}

After this paper was accepted for publication, Richard Askey pointed out to us that Theorem 1.1 had been proved in a different way by Cris T. Roosenraad in his (unpublished) Ph.D. Thesis, 1969. He used expansions with respect to generalized Hermite polynomials.

\section{REFERENCES}

1. M. Abramowitz, I.A. Stegun: Handbook of Mathematical Functions. Dover Publ. 1990.

2. W.R. Bloom, H. Heyer: Harmonic Analysis of Probability Measures on Hypergroups. De Gruyter 1995. MR 96a:43001

3. V.M. Bukhstaber, G. Felder, A.P. Veselov: Elliptic Dunkl operators, root systems, and functional equations. Duke Math. J. 76, 885-911 (1994). MR 96b:39014

4. D.L. Donoho, P.B. Stark: Uncertainty principles and signal recovery. SIAM J. Appl. Math. 49, 906 - 931 (1989). MR 90c:42003

5. C. F. Dunkl: Differential-difference operators associated to reflection groups. Trans. Amer. Math. Soc. 311, 167-183 (1989). MR 90k:33027

6. C. F. Dunkl: Integral kernels with reflection group invariance. Can. J. Math. 43, 1213-1227 (1991). MR 93g:33012

7. C. F. Dunkl: Hankel transforms associated to finite reflection groups. Contemp. Math. 138, 123 - 138 (1992). MR 94g:33011

8. H. Dym, H.P. McKean: Fourier Series and Integrals. Academic Press 1972. MR 56:945

9. M.F.E. de Jeu: The Dunkl transform. Invent. Math. 113, 147-162 (1993). MR 94m:22011 
10. M.F.E. de Jeu: An uncertainty principle for integral operators. J. Funct. Anal. 122,247 253 (1994). MR 95h:43009

11. S. Kamefuchi, Y. Ohnuki: Quantum field theory and parastatistics. University of Tokyo Press, Springer-Verlag, 1982. MR 85b:81001

12. Y. Ohnuki, S. Watanabe: Selfadjointness of the operators in Wigner's commutation relations. J. Math. Phys. 33 (11), 3653-3665 (1992). MR 93h:81065

13. E.M. Opdam: Dunkl Operators, Bessel Functions and the discriminant of a finite Coxeter group. Compos. Math. 85, 333-373 (1993). MR 95j:33044

14. M. Rösler: Convolution algebras which are not necessarily positivity-preserving. Contemp. Math. 183, 299-318 (1995). MR 96c:43006

15. M. Rösler: On the dual of a commutative signed hypergroup. Manuscr. Math. 88, 147-163 (1995). MR 96j:43004

16. M. Rösler: Bessel-type signed hypergroups on $\mathbb{R}$. In: Probability Measures on Groups and Related Structures (Proc. Conf., Oberwolfach, 1994, Ed.: H. Heyer, A. Mukherjea), pp. 292 - 304, World Scientific 1995. MR 97j:43004

17. M. Rösler, M. Voit: An uncertainty principle for ultraspherical expansions. J. Math. Anal. Appl. 1997, to appear. CMP 97:15

18. M. Rosenblum: Generalized Hermite polynomials and the Bose-like oscillator calculus. In: Operator Theory: Advances and Applications, Vol. 73, pp. 369 - 396, Birkhäuser Verlag 1994. MR 96b:33005

19. R.S. Strichartz: Uncertainty principles in harmonic analysis. J. Funct. Anal. $84,97-114$ (1989). MR 91a:42017

20. M. Voit: An uncertainty principle for commutative hypergroups and Gelfand pairs. Math. Nachr. 164, 187 - 195 (1993). MR 95d:43005

21. S. Watanabe: Sobolev type theorems for an operator with singularity. Proc. Amer. Math. Soc. 125, 129-136 (1997). MR 97c:47044

22. E.T. Whittaker, G.N. Watson: A Course of Modern Analysis. Cambridge University Press, 1935.

Mathematisches Institut, Technische Universität München, Arcisstr. 21, 80333 MünCHEN, Germany

E-mail address: roesler@mathematik.tu-muenchen.de

Mathematisches Institut, Universität Tübingen, Auf Der Morgenstelle 10, 72076 Tübingen, Germany, and Department of Mathematics, University of Virginia, Kerchof Hall, Charlottesville, Virginia, 22903-3199

E-mail address: voit@uni-tuebingen.de 